\title{
The dynamical architecture and habitable zones of the planetary system 55 Cancri
}

\author{
Jianghui $\mathbf{J i}^{1,2}$, Hiroshi Kinoshita ${ }^{3}$, Lin $\mathbf{L i u}^{4}$ and Guangyu $\mathbf{L i} \mathbf{i}^{1,2}$ \\ ${ }^{1}$ Purple Mountain Observatory, Chinese Academy of Sciences, Nanjing 210008, China \\ email: jijh@pmo.ac.cn \\ ${ }^{2}$ National Astronomical Observatories, Chinese Academy of Sciences, Beijing 100012, China \\ ${ }^{3}$ National Astronomical Observatory, Mitaka, Tokyo 181-8588, Japan \\ ${ }^{4}$ Department of Astronomy, Nanjing University, Nanjing 210093, China
}

\begin{abstract}
We performed numerical simulations to study the secular orbital evolution and dynamical structure of the quintuplet planetary system 55 Cancri using the self-consistent orbital solutions of Fischer et al. (2008).
\end{abstract}

The nearby star 55 Cancri is of spectral type $\mathrm{K} 0 / \mathrm{G} 8 \mathrm{~V}$ with a mass of $0.92 \pm 0.05 \mathrm{M}_{\odot}$. Two giant planets are reported to be trapped in a 3:1 orbital resonance and Fischer et al. (2008) revealed a fifth planet in this system. Herein, we report on simulations of the secular evolution and dynamical structure of this system.

We show that this system can be stable for at least $10^{8} \mathrm{yr}$. In addition, we extensively studied the planetary configuration of four outer companions with one terrestrial planet in the wide region 0.790-5.900 AU to examine the existence of a potential asteroidal structure and Habitable Zones (HZs). We find that there are unstable regions for the orbits about 4:1, 3:1 and 5:2 mean motion resonances (MMRs) with the outermost planet in the system, and several stable orbits can remain at 3:2 and 1:1 MMRs, a configuration which bears some resemblance with the asteroidal belt in the solar system.

From a dynamical point of view, candidate HZs for the existence of more potential terrestrial planets reside in the range between 1.0 AU and 2.3 AU for relatively low eccentricities. Moreover, our numerical simulations suggest that additional Earth-like planets can coexist with the five known planets in this system over secular timescale. This result suggests that abundant measurements and space missions (e.g. SIM Lite) should focus on this system in the future. The detailed results of this work may be found in Ji et al. (2009).

\section{Acknowledgements}

This work is financially supported by the National Natural Science Foundations of China (Grants 10973044, 10833001, 10573040, 10673006), NSF of Jiangsu Province, and the Foundation of Minor Planets of Purple Mountain Observatory.

\section{References}

Fischer, D. A., Marcy, G. W., Butler, R. P. et al. 2008, ApJ 675, 790

Ji, J., Kinoshita, H., Liu, L., \& Li, G. 2009, Res. Astron. \&3 Astrophys. 9, 703 